\title{
ON THE TOPOLOGICAL EXTENSION TO THE BOUNDARY OF BIHOLOMORPHIC MAPS IN $\mathrm{C}^{n}\left({ }^{1}\right)$
}

BY

\author{
R. MICHAEL RANGE
}

\begin{abstract}
Let $F: D_{1} \rightarrow D_{2}$ be a biholomorphic map between bounded domains in $\mathrm{C}^{n}$ with piecewise smooth strictly pseudoconvex boundaries. It is shown that $F$ is Hölder continuous of some positive order, and hence $F$ extends to a homeomorphism of the closures of the domains. This generalizes recent results of G. M. Henkin and N. Vormoor for domains with smooth strictly pseudoconvex boundary.
\end{abstract}

Introduction. Recently Henkin [10] and Vormoor [16] proved independently the following higher dimensional version of a classical result in the complex plane: a biholomorphic map between two bounded domains in $\mathbf{C}^{n}$ with smooth, strictly pseudoconvex boundaries extends to a homeomorphism of the closure of the domains. Both proofs, although quite different from one another in many aspects, have one fundamental idea in common: they make use of the invariance of the Carothéodory metric under biholomorphic maps.

Henkin's method seems to be more powerful, as he can show that such a biholomorphic map is actually Hölder continuous of order $1 / 2$.

Most recently Fefferman [4], by a different method, was able to prove that biholomorphic maps between strictly pseudoconvex domains with $C^{\infty}$ boundary extend as $C^{\infty}$ maps to the boundary.

In this paper we generalize the result of Henkin and Vormoor to domains with piecewise smooth strictly pseudoconvex boundaries (see $\S 1.7$ for the precise definition). We show that a biholomorphic map between two such domains is Hölder continuous of some positive order which depends on the minimal angle at the corners of the domains. The proof combines the techniques of Henkin and Vormoor with a careful analysis of the situation at the corners.

One of the key steps of the proof involves an estimate from below for the Carathéodory metric. At the local level, this estimate is reduced to the case of smooth boundaries by using the monotonicity of the metric; the further reduction to the case of a ball is elementary, though quite delicate. In order to pass

Received by the editors November 4, 1974.

AMS (MOS) subject classifications (1970). Primary 32H99; Secondary 32H15, 32A30.

(1)Research supported by a fellowship of the Research Foundation of the State University of New York. 
to the global level one adapts the techniques of Hörmander [11] and Diederich [3] to the case considered here. By using the uniform estimates for solutions of the $\bar{\partial}$-equation (cf. Range and Siu [14]), I had obtained a simple proof of the required approximation theorem (Proposition 3.1). After completion of this manuscript, I received a preprint of [7], in which a similar proof is given for the case of smooth boundaries. I thus decided to omit the proof in this paper and refer the reader to [7] instead.

For domains with smooth strictly pseudoconvex boundary more precise estimates for the Carathéodory metric have been obtained by Henkin [10] and Graham [6] , [7].

Another basic step is an estimate for the distance to the boundary under biholomorphic maps (Theorem 4.11), which may be of independent interest. It is this result which reflects most deeply the geometry of the domains near the corners. Part of the proof is based on an analogue of Schwarz's Lemma for sectors of a disc.

For a summary of the contents of the various paragraphs the reader is referred to the opening statements of each paragraph. $\S 6$ contains some open questions which arise naturally from the work in this paper.

1. Preliminaries. In this paragraph we introduce some notations, discuss the basic properties of the Carathéodory metric and define precisely the domains which are considered in this paper.

(1.1) For a point $z=\left(z_{1}, \ldots, z_{n}\right)$ in $\mathbf{C}^{n}$, we set

$$
|z|=\left(\sum_{i=1}^{n}\left|z_{i}\right|^{2}\right)^{1 / 2} \text {. }
$$

For $a \in \mathbf{C}^{n}, r>0, B(a, r)=\left\{z \in \mathbf{C}^{n}:|z-a|<r\right\}$ denotes the open ball with center $a$ and radius $r$. For $M \subset \mathbf{C}^{n}$ and $z \in \mathbf{C}^{n}$,

$$
d(z, M)=\inf \{|z-P|: P \in M\}
$$

denotes the Euclidean distance from $z$ to $M$.

The natural pairing between a cotangent vector $\alpha$ and a tangent vector $v$ is denoted by $\langle\alpha, v\rangle$. In particular, if $f$ is a $C^{1}$ function near a point $z$, and $v=$ $\left(v_{1}, \ldots, v_{n}\right) \in \mathbf{C}^{n}$, then

$$
\langle\partial f(z), v\rangle=\sum_{i=1}^{n}\left(\partial f / \partial z_{i}\right)(z) v_{i}
$$

(1.2) For a complex valued function $f$ defined on a set $D \subset \mathbf{C}^{n}$, the supremum norm of $f$ in $D$ is denoted by 


$$
\|f\|_{D}=\sup \{|f(z)|: z \in D\}
$$

For a $(0,1)$ form $\alpha=\Sigma \alpha_{i} d \bar{z}_{i},\|\alpha\|_{D}=\max \left\{\left\|\alpha_{i}\right\|_{D}: 1 \leqslant i \leqslant n\right\}$. When no confusion can arise, we may drop the subscript $D$. No other norms for function spaces are used in this paper.

For an open set $D \subset \mathbf{C}^{n}$, the space of holomorphic functions on $D$ is denoted by $O(D)$. We introduce two subclasses of $O(D)$ :

$$
F(D)=\left\{f \in O(D):\|f\|_{D} \leqslant 1\right\},
$$

and, for $P \in D$,

$$
F_{P}(D)=\{f \in F(D): f(P)=0\} .
$$

(1.3) In order to avoid introducing many irrelevant constants, we use the following notation. If $A, B$ are functions of a variable $x, A \lesssim B$ means that there is a constant $\gamma$, such that $A(x) \leqslant \gamma B(x)$ for all $x$ under consideration. If $A$ and $B$ depend also on some parameter $\gamma, A \lesssim B$ uniformly in $\lambda$ means that the constant $\gamma$ can be chosen independently of $\lambda$. Finally $A \sim B$ means that $A \lesssim B$ and $B \lesssim A$.

(1.4) For a bounded domain $D$ in $\mathrm{C}^{n}$, the Carathéodory distance $d_{C}: D \times$ $D \rightarrow \mathrm{R}$ is defined by

$$
d_{C}\left(z_{1}, z_{2}\right)=\sup \left\{\rho\left(f\left(z_{1}\right), f\left(z_{2}\right)\right): f \in F(D)\right\},
$$

where $\rho$ is the Poincare metric on the open unit disc (cf. Behnke and Thullen [2, Chapter VII, §8]). In this paper we work with the infinitesimal form $C_{D}(z, v)$ of the Carathéodory distance of a domain $D$. For each $z \in D, C_{D}(z, \cdot)$ is the function defined on the complex tangent space of $D$ at $z$ by

$$
C_{D}(z, v)=\sup \{|\langle\partial f(z), v\rangle|: f \in F(D)\}
$$

(cf. Reiffen [15]). It is easy to see that

$$
\sup \{|\langle\partial f(z), v\rangle|: f \in F(D)\}=\sup \left\{|\langle\partial f(z), v\rangle|: f \in F_{z}(D)\right\} .
$$

We call the function $C_{D}$ the Carathéodory metric of the domain $D$.

The Carathéodory distance $d_{C}$ of $D$ is related to its infinitesimal form $C_{D}$ as follows. Let $\phi:[a, b] \rightarrow D$ be a differentiable curve; its length $L(\phi)$ with respect to $d_{C}$ is defined by

$$
L(\phi)=\sup \sum_{\nu=1}^{r} d_{C}\left(\phi\left(t_{\nu}\right), \phi\left(t_{\nu-1}\right)\right),
$$

where the supremum is taken over all finite partitions $a=t_{0}<t_{1}<\cdots<t_{r}=$ $b$ of $[a, b]$. One has 


$$
L(\phi)=\int_{a}^{b} C_{D}\left(\phi(t), \phi^{\prime}(t)\right) d t
$$

(cf. Reiffen [15]). This result will not be needed in this paper.

The following property of the Carathéodory metric is fundamental.

1.5. Lemma. Suppose $F: D_{1} \rightarrow D_{2}$ is a holomorphic map between two bounded domains in $\mathbf{C}^{n}$. Then

$$
C_{D_{2}}\left(F(z), F_{* z} v\right) \leqslant C_{D_{1}}(z, v)
$$

for all $z \in D_{1}, v \in \mathbf{C}^{n}$. In particular, equality holds if $F$ is biholomorphic. ( $F_{* z}$ denotes the Jacobian matrix of $F$ at $z$.)

The proof is straightforward.

By applying this lemma to an inclusion map, one obtains

1.6. LEMMA. The Carathéodory metric is a monotonically decreasing function of the domain, i.e., for $D_{1} \subset D_{2}$,

$$
C_{D_{2}}(z, v) \leqslant C_{D_{1}}(z, v)
$$

for all $z \in D_{1}$ and $v \in \mathbf{C}^{n}$.

(1.7) A bounded domain $D$ in $\mathbf{C}^{n}$ is said to have a piecewise smooth boundary if there exist

(i) a finite open covering $\left\{U_{j}\right\}_{j=1}^{k}$ of an open neighborhood $U$ of $\partial D$, and

(ii) $C^{1}$ functions $\rho_{j}: U_{j} \rightarrow \mathbf{R}(1 \leqslant j \leqslant k)$, such that

(a) $D \cap U=\left\{x \in U\right.$ : for $1 \leqslant j \leqslant k$, either $x \notin U_{j}$ or $\left.\rho_{j}(x)<0\right\}$,

(b) for $1 \leqslant i_{1}<\cdots<i_{l} \leqslant k$, the 1 -forms $d \rho_{i_{1}}, \ldots, d \rho_{i_{l}}$ are linearly independent over $\mathbf{R}$ at every point $x \in \bigcap_{\nu=1}^{l} U_{i_{\nu}}$.

We call $\left\{U_{j}, \rho_{j}\right\}_{j=1}^{k}$ a frame for $D$.

For $1 \leqslant j \leqslant k$, define $S_{j}=\left\{x \in U_{j}: \rho_{j}(x)=0\right\}$.

$D$ is said to have piecewise smooth strictly pseudoconvex boundary, if there is a frame $\left\{U_{j}, \rho_{j}\right\}_{j=1}^{k}$ for $D$, such that, for $1 \leqslant j \leqslant k$, the function $\rho_{j}$ is $C^{2}$ and strictly plurisubharmonic on $U_{j}$. We note that any sufficiently small $C^{2}$ perturbation of the functions $\rho_{j}, 1 \leqslant j \leqslant k$, gives again a domain with piecewise smooth strictly pseudoconvex boundary.

2. Local estimates from below. In this paragraph we establish an estimate from below in terms of the distance to the boundary for the Carathéodory metric on "small" sets near the boundary. The precise result is as follows.

2.1. Proposition. Let $D$ be a domain with piecewise smooth strictly pseudoconvex boundary. For each $z_{0} \in \partial D$ there is a ball $B=B\left(z_{0}, \epsilon\right)$ such that for all $z \in B \cap D$ and $v \in \mathbf{C}^{n}$ 


$$
C_{B \cap D}(z, v) \gtrsim|v| d(z, \partial D)^{-1 / 2} .
$$

The proof of this estimate consists of a stepwise reduction to the case of a ball. For smooth strictly pseudoconvex boundaries more precise estimates have been obtained by Henkin [10] and Graham [6], [7]. We give a simplified proof for the case we are interested in. The proof will involve a series of lemmas.

2.2. LemmA. If $B_{r}$ is a ball of radius $r$, then

$$
C_{B_{r}}(z, v) \gtrsim|v| d\left(z, \partial B_{r}\right)^{-1 / 2}
$$

for all $z \in B_{r}$ and $v \in \mathbf{C}^{n}$.

PROoF. By reducing the problem to the case of a disc and explicit calculations one obtains

$$
C_{B_{r}}(z, v)=\left[\frac{|v|^{2}}{r^{2}-|z|^{2}}+\frac{|(z, v)|^{2}}{\left(r^{2}-|z|^{2}\right)^{2}}\right]^{1 / 2}
$$

(see, for example, the proof of Satz 5 in [15]). Since for $z \in B_{r}, r^{2}-|z|^{2} \leqslant$ $2 r(r-|z|)$, the desired estimate follows.

2.3. Lemma. Let $W$ be an open neighborhood of 0 in $\mathbf{C}^{n}$. Suppose $\rho \in$ $C^{2}(W)$ is real valued, $\rho(0)=0, d \rho(z) \neq 0$ for all $z \in W$, and the Hessian of $\rho$ is positive definite at all points of $W$. Let $M=\{z \in W: \rho(z)=0\}$. Then there is a neighborhood $V \subset W$ of 0 , such that for all $z \in V^{-}=\{z \in V: \rho(z)<0\}$ one has

$$
C_{V^{-}}(z, v) \gtrsim|v| d(z, M)^{-1 / 2} .
$$

Proof. For $p \in M$, let $n_{p}$ be the unit normal to $M$ at $p$ pointing to the side $\rho<0$. The strict convexity of $\rho$ implies that there are positive constants $r$ and $\delta$ such that $B(0,2 \delta) \subset W$ and for each $p \in B(0,2 \delta) \cap M$, if we set $B_{p}=$ $B\left(p+r n_{p}, r\right)$, then

(i) $p \in \partial B_{p}$,

(ii) $B(0,2 \delta) \cap\{z \in W: \rho(z)<0\} \subset B_{p}$.

Let $V=B(0, \delta)$. Given $z \in V^{-}$, choose $p \in M \cap B(0,2 \delta)$ such that $d(z, M)=$ $|z-p|=d\left(z, \partial B_{p}\right)$. By the monotonicity of the Carathéodory metric (Lemma 1.6) and Lemma 2.2 one obtains

$$
C_{V^{-}}(z, v) \geqslant C_{B_{p}}(z, v) \gtrsim|v| d\left(z, \partial B_{p}\right)^{-1 / 2}=|v| d(z, M)^{-1 / 2} \text {. Q.E.D. }
$$

2.4. Lemma. Let $U$ be open in $\mathbf{C}^{n}$. Suppose $\rho \in C^{2}(U)$ is strictly plurisubharmonic and $d \rho(z) \neq 0$ for all $z \in U$. Let $S=\{z \in U: \rho(z)=0\}$. Then for each $z_{0} \in S$ there is a ball $B\left(z_{0}, \epsilon\right)$ such that 


$$
C_{B\left(z_{0}, \epsilon\right) \cap\{\rho<0\}}(z, v) \gtrsim|v| d(z, S)^{-1 / 2}
$$

for all $z \in B\left(z_{0}, \epsilon\right) \cap\{\rho<0\}$ and $v \in \mathbf{C}^{n}$.

Proof. Choose $\epsilon$ so that $B\left(z_{0}, 2 \epsilon\right) \subset U$ and so that there is a biholomorphic map $T: B\left(z_{0}, 2 \epsilon\right) \rightarrow W$ onto an open neighborhood $W$ of 0 in $\mathbf{C}^{n}$ with the following properties:

(i) the (real) Hessian of $\rho \circ T^{-1}$ is positive definite on $W$;

(ii) $T\left(B\left(z_{0}, \epsilon\right)\right)$ is contained in the neighborhood $V$ given by Lemma 2.3 applied to $W$ and $\rho \circ T^{-1}$;

(iii) the Jacobian determinant of $T$ is bounded from above and below in $B\left(z_{0}, 2 \epsilon\right)$.

The map $T$ is obtained as follows. After an affine change of coordinates we can assume that $z_{0}=0$ and $\partial \rho\left(z_{0}\right)=(1,0, \ldots, 0)$. Then the inverse $z=$ $T^{-1}(w)$ of $T$ is defined by the equations

$$
z_{1}=w_{1}-\frac{1}{2} \sum_{\nu, \mu} \frac{\partial^{2} \rho}{\partial z_{\nu} \partial z_{\mu}}(0) w_{\nu} w_{\mu}, \quad z_{\mu}=w_{\mu}, \quad \mu=2, \ldots, n .
$$

By the chain rule, $\partial^{2}\left(\rho \circ T^{-1}\right)(0) / \partial w_{\nu} \partial w_{\mu}=0$ for $\mu, \nu=1, \ldots, n$, which implies (i) at 0 , and hence in a neighborhood of 0 ; (ii) and (iii) are trivial.

Now if $z \in B^{-}=B\left(z_{0}, \epsilon\right) \cap\{\rho<0\}$, one obtains, by Lemmas 1.5 and 1.6,

$$
\begin{aligned}
C_{B^{-}}(z, v) & =C_{T\left(B^{-}\right)}\left(T(z), T_{* z} v\right) \geqslant C_{V}-\left(T(z), T_{*_{z}} v\right) \\
& \gtrsim\left|T_{* z} v\right| d\left(T(z), T\left(B\left(z_{0}, 2 \epsilon\right) \cap S\right)\right)^{-1 / 2} \\
& \gtrsim|v| d\left(z, B\left(z_{0}, 2 \epsilon\right) \cap S\right)^{-1 / 2}=|v| d(z, S)^{-1 / 2},
\end{aligned}
$$

where the last two inequalities follow from Lemma 2.3 and (iii) above. Q.E.D.

(2.5) We can now conclude the proof of Proposition 2.1. Let $\left\{U_{j}, \rho_{j}\right\}_{j=1}^{k}$ be a frame for $D$. After renumbering, we can assume that $z_{0} \in S_{j}$ for $j=1$, $\ldots, l$ and $z_{0} \notin S_{j}$ for $j=l+1, \ldots, k$. By applying Lemma 2.4 to $U_{j}$ and $\rho_{j}$, one obtains $\epsilon_{j}>0$ for $j=1, \ldots, l$, such that

$$
C_{B\left(z_{0}, \epsilon_{j}\right) \cap\left\{\rho_{j}<0\right\}}(z, v) \gtrsim|v| d\left(z, S_{j}\right)^{-1 / 2} .
$$

Let $\epsilon=\min _{1 \leqslant j \leqslant l} \epsilon_{j}$. By (1.6)

$$
C_{B\left(z_{0}, \epsilon\right) \cap D}(z, v) \gtrsim|v| d\left(z, S_{j}\right)^{-1 / 2}
$$

for all $z \in B\left(z_{0}, \epsilon\right) \cap D$ and $j=1, \ldots, l$. Since in a neighborhood of $z_{0}$, $d(z, \partial D)=\min _{1 \leqslant j \leqslant l} d\left(z, S_{j}\right)$, the conclusion follows.

3. The global estimate. The technique for passing from the local estimate of $\S 2$ to an estimate for the Carathéodory metric on the whole domain goes 
back, in case of the Bergman metric, to Hörmander [11, Lemma 3.5.2] and was later modified by Diederich [3]. For the case considered here, the main ingredient is the following approximation result, whose proof is essentially due to Diederich.

3.1. Proposition. Let $D$ be a domain with piecewise smooth strictly pseudoconvex boundary. Let $z_{0} \in \partial D$ and $\epsilon>0$ be given. Then, for all $\eta>0$ and sufficiently small $\delta>0$, there is a constant $L=L(\delta, \eta)$ such that, given $f \in$ $F\left(B\left(z_{0}, \epsilon\right) \cap D\right)$ and $\zeta \in B\left(z_{0}, \delta\right) \cap D$ there is $F \in O(D)$ which satisfies

(i) $\|F-f\|_{B\left(z_{0}, \delta\right) \cap D}<\eta$;

(ii) $F(\zeta)=f(\zeta), \partial F(\zeta)=\partial f(\zeta)$;

(iii) $\|F\|_{D} \leqslant L$.

A simple proof for domains with smooth strictly pseudoconvex boundary, which uses a modification of Hörmander's technique and the uniform estimates for solutions of the $\bar{\partial}$-equation, is given in [7, Theorem 2]. This proof carries over to the domains considered here; the required estimates for the $\bar{\partial}$-equation for this case are proved in Range and Siu [14].

3.2. Theorem. Let $D$ be a domain with piecewise smooth strictly pseudoconvex boundary. Then there is $\epsilon>0$ such that for all $z \in D$ with $d(z, \partial D)<\epsilon$ and $v \in \mathbf{C}^{n}$

$$
C_{D}(z, v) \gtrsim|v| d(z, \partial D)^{-1 / 2}
$$

Proof. By compactness of $\partial D$ we can cover $\partial D$ by finitely many open balls $B\left(z_{\lambda}, \epsilon_{\lambda}\right), \lambda=1, \ldots, m$, with centers $z_{\lambda} \in \partial D$, such that Proposition 2.1 is valid for each $B\left(z_{\lambda}, \epsilon_{\lambda}\right)$, and if $B\left(z_{\lambda}, \delta_{\lambda}\right)$ is a ball with the properties described in Proposition 3.1 applied to $B\left(z_{\lambda}, \epsilon_{\lambda}\right)$, then $\partial D \subset \bigcup_{\lambda=1}^{m} B\left(z_{\lambda}, \delta_{\lambda}\right)$. Fix $\eta>0$ and let $L_{\lambda}=L\left(\eta, \delta_{\lambda}\right)$ be the constant given by Proposition 3.1. Let $L=$ $\max _{1 \leqslant \lambda \leqslant m} L_{\lambda}$, and choose $\epsilon>0$ such that $\{z \in D: d(z, \partial D)<\epsilon\} \subset$ $\bigcup_{\lambda=1}^{m} B\left(z_{\lambda}, \delta_{\lambda}\right)$. Proposition 3.1 implies that for $z \in B\left(z_{\lambda}, \delta_{\lambda}\right) \cap D$

$$
C_{D}(z, v) \geqslant(1 / L) C_{B\left(z_{\lambda}, \delta_{\lambda}\right) \cap D}(z, v) .
$$

Moreover, by Lemma 1.6 and Proposition 2.1,

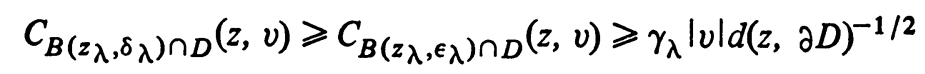

for some constant $\gamma_{\lambda}>0$ and each $\lambda=1, \ldots, m$. Setting $\gamma=\min _{1 \leqslant \lambda \leqslant m} \gamma_{\lambda}$, (*) and (**) imply

$$
C_{D}(z, v) \geqslant(\gamma / L)|v| \cdot d(z, \partial D)^{-1 / 2}
$$

for all $z \in D$ with $d(z, \partial D)<\epsilon$. Q.E.D.

REMARK. By introducing a peaking function at $z_{0} \in \partial D$ and arguing as in 
Diederich $[3, \S 6]$, one can prove the following sharper relationship between the local and global Carathéodory metric (see also [6]).

3.3. Proposition. Let $D$ be as in (3.2) and $z_{0} \in \partial D$. For any neighborhood $V$ of $z_{0}$ one has

$$
\lim _{z \rightarrow z_{0} ; z \in D \cap V} \frac{C_{D \cap V}(z, v)}{C_{D}(z, v)}=1
$$

for all $v \in \mathbf{C}^{n}$.

4. Estimates for the distance to the boundary. In order to study the behavior of the distance to the boundary under biholomorphic maps, we follow an idea of Vormoor [16] and relate the distance to the boundary to an auxiliary function which transforms in an obvious way under such maps.

4.1. Definition. Let $D$ be a bounded domain in $\mathbf{C}^{n}$ and $P \in D$. For $z \in$ $D$, define

$$
h_{D, P}(z)=\sup _{f \in F_{P}(D)}|f(z)| .
$$

$h_{D, P}(z)$ is closely related to the Carathéodory distance between $P$ and $z$. By using the invariance of the Poincare metric on the unit disc, one easily obtains

$$
d_{C}(P, z)=\frac{1}{2} \cdot \log \frac{1+h_{D, P}(z)}{1-h_{D, P}(z)} .
$$

4.2. LemMA. If $D_{1}$ and $D_{2}$ are bounded domains in $\mathbf{C}^{n}$ and $F: D_{1} \rightarrow D_{2}$ is a holomorphic map, then

$$
h_{D_{2}, F(P)}(F(z)) \leqslant h_{D_{1}, P}(z) \quad \text { for all } z \in D .
$$

If $F$ is biholomorphic, equality holds.

The proof is trivial.

4.3. Proposition. Let $D$ be a domain with piecewise smooth strictly pseudoconvex boundary, and let $P \in D$. Then

$$
1-h_{D, P}(z) \lesssim d(z, \partial D) \quad \text { for } z \in D .
$$

In case $\partial D$ is $C^{\infty}$ and strictly pseudoconvex, this is Satz 2 in Vormoor [16]; the idea of the proof given there carries over to the more general case. The main tool required is the following parametrized version of the well-known construction of "peaking functions" for strictly pseudoconvex domains (cf. Gunning and Rossi [8, Corollary IX, C7], Henkin [9] and Ramirez [13] ; for the construction in case of $C^{2}$ boundaries, see also Range and Siu [14]). 
4.4. THEOREM. Let $D$ be a domain with piecewise smooth strictly pseudoconvex boundary with frame $\left\{U_{j}, \rho_{j}\right\}_{j=1}^{k}$. For each $j=1, \ldots, k$ there is a function $F_{j}(\zeta, z): U_{j} \times W \rightarrow \mathbf{C}$, where $W$ is a neighborhood of $\bar{D}$, such that

(a) $F_{j} \in C^{1}\left(U_{j} \times W\right)$ and is holomorphic in $z$;

(b) $F_{j}(\zeta, \zeta)=1$ for $\zeta \in \partial D \cap\left\{\rho_{j}=0\right\}$;

(c) $\left|F_{j}(\zeta, z)\right|<1$ for $\zeta \in \partial D \cap\left\{\rho_{j}=0\right\}$ and $z \in \bar{D}-\{\zeta\}$.

The proof of (4.3) is now quite easy. By (c), $\left|F_{j}(\zeta, P)\right|<1$ for $\zeta \in \partial D \cap$ $\left\{\rho_{j}=0\right\}$, and hence, after shrinking $U_{j}$, we can assume that

$$
\left|F_{j}(\zeta, P)\right| \leqslant q<1 \quad \text { for } \zeta \in U_{j} \text { and } j=1, \ldots, k \text {. }
$$

For $j \in\{1, \ldots, k\}$ and $\zeta \in U_{j}$, let $\phi_{j, \zeta}$ be the automorphism of the unit disc $\Delta$, which takes the point $F_{j}(\zeta, P)$ into 0 and 1 into 1 . By $(*),\left|\phi_{j, \zeta}^{\prime}(w)\right| \leqslant K_{1}<$ $\infty$ for $w \in \bar{\Delta}$ and for all $j$ and $\zeta$ under consideration. Together with (a) it follows that $\phi_{j, \zeta^{\circ}} F_{j}(\zeta, \cdot) \in F_{P}(D)$ and that its first order partial derivatives with respect to $z$ are bounded on $D$ by a constant $K$ which is independent of $j \in\{1, \ldots, k\}$ and $\zeta \in \partial D \cap\left\{\rho_{j}=0\right\}$. Given $z \in D$, choose $\zeta \in \partial D$ such that $|z-\zeta|=d(z, \partial D)$, and $j \in\{1, \ldots, k\}$ such that $\rho_{j}(\zeta)=0$. Then

$$
\begin{aligned}
1-h(z) & \leqslant 1-\left|\phi_{j, \zeta} \circ F_{j}(\zeta, z)\right| \leqslant\left|\phi_{j, \zeta} \circ F_{j}(\zeta, \zeta)-\phi_{j, \zeta} \circ F_{j}(\zeta, z)\right| \\
& \leqslant n K|\zeta-z|=n K d(z, \partial D) \text {. Q.E.D. }
\end{aligned}
$$

We now estimate $d(z, \partial D)$ from above.

4.5. Proposition. Let $D$ be a bounded domain with piecewise smooth boundary and let $P \in D$. Then there is an integer $m>0$ and a constant $\epsilon>0$ such that

$$
d(z, \partial D)^{m} \lesssim 1-h_{D, p}(z) \text { for } z \in D, \quad d(z, \partial D)<\epsilon .
$$

If $D$ has smooth boundary, $m$ can be chosen to be 1 .

REMARK. In case $D$ has smooth boundary, this is Satz 4 in Vormoor [16]. However, there seems to be a gap in $\S 5$ of the proof given there [16, p. 244]

In order to prove the proposition we need a geometric lemma. For $\zeta \in \mathbf{C}^{n}$, $v$ a unit vector in $\mathbf{C}^{n}, 0<\alpha<\pi$ and $\gamma>0$, we denote by $C(\zeta, v, \alpha, \gamma)$ the open cone with vertex $\xi$, axis in the direction of $v$, aperture $\alpha / 2$ and height $\gamma$.

4.6. Lemma. Let $D$ be a domain with piecewise smooth boundary. Then there are constants $\epsilon>0, \gamma>0$ and $0<\alpha<\pi$ such that the following holds. For each $z \in D$ with $d(z, \partial D)<\epsilon$ there is $\zeta(z) \in \partial D$ and a unit vector $v(z) \in$ $\mathrm{C}^{n}$ such that $z$ lies on the axis of the cone $C(\zeta(z), v(z), \alpha, \gamma)$ and

(i) $C(\zeta(z), v(\dot{z}), \alpha, \gamma) \subset D$,

(ii) $d(z, \partial D) \sim|z-\zeta(z)|$. 
Proof. Let $\left\{U_{j}, \rho_{j}\right\}_{j=1}^{k}$ be a frame for $D$. Suppose $z_{0} \in \partial D$ and that, after renumbering, $\rho_{j}\left(z_{0}\right)=0$ for $j=1, \ldots, l$ and $\rho_{j}(z) \neq 0$ for $j=l+1, \ldots$, $k$. Choose $B\left(z_{0}, \epsilon^{\#}\right)$ such that $\rho_{j}(z) \neq 0$ for $z \in B\left(z_{0}, \epsilon^{\#}\right)$ and $j>l$. Let $\tilde{v}=$ $-\Sigma_{j=1}^{l} \operatorname{grad} \rho_{j}\left(z_{0}\right)$ and $v=\tilde{v} /|\tilde{v}|$. Note that $\tilde{v} \neq 0$, since $\operatorname{grad} \rho_{1}\left(z_{0}\right), \ldots$, grad $\rho_{l}\left(z_{0}\right)$ are linearly independent (cf. (1.7) (b)). Now choose $\delta>0$ and $\gamma>$ 0 , such that

$$
B\left(z_{0}, \delta\right) \cap D \subset \bigcup_{\zeta \in B\left(z_{0}, \epsilon \# / 2\right) \cap \partial D}\{z: z=\zeta+\lambda v, 0<\lambda \leqslant \gamma\} \subset D .
$$

Since $v$ is not tangent to $S_{j}$ near $z_{0}$ for $j=1, \ldots, l$, one can choose $0<\alpha<$ $\pi$ so that for $\zeta \in B\left(z_{0}, \epsilon^{\#} / 2\right) \cap \partial D$ the cone $C=C(\zeta, v, \alpha, \gamma) \subset D$.

Given $z \in B\left(z_{0} \delta\right) \cap D$, choose $\zeta=\zeta(z) \in B\left(z_{0}, \epsilon^{\#} / 2\right) \cap \partial D$ such that $z=$ $\zeta(z)+\lambda v$ for some $0<\lambda \leqslant \gamma$. Since $C(\zeta(z), v, \alpha, \gamma) \subset D$

$$
|z-\zeta(z)| \lesssim d(z, \partial D) \leqslant|z-\zeta(z)| .
$$

This proves the lemma for all $z \in B\left(z_{0}, \delta\right) \cap D$. To prove the general case, cover $\partial D$ by finitely many such balls $B\left(z_{\lambda}, \delta_{\lambda}\right)$ and choose $\epsilon>0$ so that $\{z \in D: d(z, \partial D)<\epsilon\} \subset \bigcup B\left(z_{\lambda}, \delta_{\lambda}\right)$. Q.E.D.

We now come to the proof of Proposition 4.5. Let $\alpha, \gamma, \epsilon$ be the constants given by Lemma 4.6. We may assume that $\alpha=\pi / m$, where $m$ is a positive integer. Define

$$
R=\left\{\lambda \in \mathrm{C}: \lambda=r e^{i \theta}, 0<r<\gamma,|\theta|<\alpha / 2\right\} .
$$

Let $\phi: R \rightarrow \Delta=\{w \in \mathrm{C}:|w|<1\}$ be a biholomorphic map which carries the interval $\{\lambda \in R: 0<\lambda<\gamma\}$ onto the interval $(-1,1)$ in $\Delta$ in such a way that $\phi(0)=1$ and $\phi(\gamma)=-1$ (note that $\phi$ extends to a homeomorphism $\bar{R} \rightarrow \bar{\Delta}$ ). $\phi$ can be constructed explicitly (cf. $[1$, p. 384]), and it has the following important property:

$\phi$ is holomorphic in a neighborhood $N$ of 0 , and $\phi(\lambda)=\widetilde{\phi}\left(\lambda^{m}\right)$, where $\tilde{\phi}$ is biholomorphic at 0 .

Hence we can choose $N$ and $\eta>0$ such that

$$
\left|\widetilde{\phi}^{\prime}\left(\lambda^{m}\right)\right| \geqslant \eta \text { for } \lambda \in N
$$

Let now $t \in D$ and $d(t, \partial D)<\epsilon$, and let $C(\zeta(t), v(t), \alpha, \gamma)$ be the cone given by Lemma 4.6. Define

$$
S(t)=\left\{z \in \mathbf{C}^{n}: z=\zeta(t)+\lambda v(t), \lambda \in R\right\} .
$$

Then $S(t) \subset D$, and there is $\lambda_{t}, 0<\lambda_{t}<\gamma$, such that $t=\zeta(t)+\lambda_{t} v(t)$. Moreover, by 4.6 (ii),

$$
\lambda_{t}=|t-\zeta(t)| \gtrsim d(t, \partial D) .
$$


From now on we assume that $\epsilon$ is so small that $\lambda_{t} \in N$ and $\phi\left(\lambda_{t}\right)>0$ whenever $d(t, \partial D)<\epsilon$.

Let $\Phi_{t}: \Delta \rightarrow S(t)$ be the holomorphic map given by

$$
\Phi_{t}(w)=\zeta(t)+\phi^{-1}(w) \cdot v(t) .
$$

Since $Q=\left\{\Phi_{t}(0): t \in D\right.$ and $\left.d(t, \partial D)<\epsilon\right\}$ is relatively compact in $D$, a simple normal family argument shows that there is $q<1$ such that $\|f\|_{Q} \leqslant q$ for all $f \in F_{P}(D)$. Define the automorphism $\tau_{a}, a \in \Delta$, of $\Delta$ by

$$
\tau_{a}(z)=(z-a) /(z \bar{a}-1) .
$$

The derivative of $\tau_{a}$ is bounded from above and below on $\bar{\Delta}$, and the bounds can be chosen independently of $a$ for $|a| \leqslant q$. This implies

$$
d\left(\tau_{a}(z), \partial \Delta\right) \sim d(z, \partial \Delta)
$$

for all $z \in \Delta,|a| \leqslant q$.

For arbitrary $f \in F_{P}(D)$, the function $\tau_{f \circ \Phi_{t}(0)} \circ f \circ \Phi_{t}: \Delta \rightarrow \Delta$ maps 0 into 0. Hence, by Schwarz's Lemma and (4.9)

$$
1-|w| \leqslant 1-\left|\tau_{f_{\circ} \Phi_{t}(0)} \circ f \circ \Phi_{t}(w)\right| \lesssim 1-\left|f \circ \Phi_{t}(w)\right| .
$$

For $w=\phi\left(\lambda_{t}\right)$, this gives $1-\left|\phi\left(\lambda_{t}\right)\right| \lesssim 1-|f(t)|$, and since $f$ was arbitrary, one gets

$$
1-\left|\phi\left(\lambda_{t}\right)\right| \lesssim 1-h_{D, P}(t)
$$

Finally, since $\phi\left(\lambda_{t}\right)>0$ and $\phi(0)=1$,

$$
1-\left|\phi\left(\lambda_{t}\right)\right|=\left|\phi(0)-\phi\left(\lambda_{t}\right)\right|=\left|\widetilde{\phi}(0)-\widetilde{\phi}\left(\lambda_{t}^{m}\right)\right| \geqslant \eta \cdot \lambda_{t}^{m},
$$

where the inequality follows from (4.7). The above estimate, combined with (4.8) and (4.10), completes the proof of the general case of the proposition.

In case $D$ has smooth boundary, the disc sector $S(t)$ in the above proof can be replaced by an analytic disc, and the above arguments go through with $m=1$. Q.E.D.

Combining Propositions 4.3 and 4.5, and Lemma 4.2, one obtains the main result of this paragraph.

4.11. Theorem. Let $D_{1}$ and $D_{2}$ be two bounded domains in $\mathbf{C}^{n}$ with piecewise smooth strictly pseudoconvex boundaries. Then there is a positive integer $m$, such that, if $F: D_{1} \rightarrow D_{2}$ is a biholomorphic map, then

$$
d\left(z, \partial D_{1}\right)^{m} \lesssim d\left(F(z), \partial D_{2}\right) \lesssim d\left(z, \partial D_{1}\right)^{1 / m} \quad \text { for all } z \in D_{1} .
$$


5. The Hölder continuity of biholomorphic maps. In this paragraph we prove an estimate for the growth of the Jacobian of biholomorphic maps near the boundary. This will imply the Hölder continuity of such maps. First, we prove an elementary estimate from above for the Carathéodory metric.

5.1. Lemma. Let $D$ be a bounded domain in $\mathbf{C}^{n}$. Then, for all $z \in D$ and $v \in \mathrm{C}^{n}$, one has

$$
C_{D}(z, v) \leqslant|v| d(z, \partial D)^{-1}
$$

Proof. Let $z \in D, r=d(z, \partial D)>0$, and let $v \in \mathbf{C}^{n}$ with $|v|=1$. By applying Schwarz's Lemma to the analytic disc $\left\{w \in \mathbf{C}^{n}: w=z+\lambda v,|\lambda|<r\right\}$ $\subset D$, one obtains, for an arbitrary $f \in F_{z}(D):|\langle\partial f(z), v\rangle| \leqslant 1 / r$, which clearly implies the statement of the lemma.

Now let $D_{1}, D_{2}$ be bounded domains with piecewise smooth strictly pseudoconvex boundaries, and let $F: D_{1} \rightarrow D_{2}$ be a biholomorphic map. Let $z \in D$ and $v \in \mathrm{C}^{n}$. Then $C_{D_{2}}\left(F(z), F_{* z} v\right)=C_{D_{1}}(z, v)$, by Lemma 1.5. Together with Theorem 3.2 and Lemma 5.1, this implies

$$
\left|F_{* z} v\right| d\left(F(z), \partial D_{2}\right)^{-1 / 2} \lesssim|v| d\left(z, \partial D_{1}\right)^{-1}
$$

and hence

$$
\left|F_{* z} v\right| \lesssim|v| d\left(F(z), \partial D_{2}\right)^{1 / 2} / d\left(z, \partial D_{1}\right) .
$$

Together with Theorem 4.11, this implies

$$
\left\|F_{* z}\right\| \lesssim d\left(z, \partial D_{1}\right)^{-1+1 / 2 m} \text { for all } z \in D_{1} .
$$

We now apply the following generalization of a theorem of Hardy and Littlewood; for the case of the unit disc, a proof can be found in Goluzin [5, Chapter IX, §5, Theorems 3 and 4], and the method of proof extends readily to the more general situation.

5.3. LEMMA. Let $D$ be a bounded domain in $\mathbf{R}^{n}$ with piecewise smooth boundary. Suppose $f$ is a $C^{1}$ function on $D$, such that, for some $0<\alpha \leqslant 1$, $|\operatorname{grad} f(x)| \lesssim d(x, \partial D)^{\alpha-1}$ for $x \in D$. Then, for all $x_{1}, x_{2} \in D$,

$$
\left|f\left(x_{1}\right)-f\left(x_{2}\right)\right| \lesssim\left|x_{1}-x_{2}\right|^{\alpha}
$$

(5.2) and (5.3) now prove the main result of this paper.

5.4. THEOREM. Let $D_{1}$ and $D_{2}$ be bounded domains in $\mathbf{C}^{n}$ with piecewise smooth strictly pseudoconvex boundaries. Then there is $\alpha>0$, such that, if $F$ : $D_{1} \rightarrow D_{2}$ is a biholomorphic map, then 


$$
\left|F\left(z_{1}\right)-F\left(z_{2}\right)\right| \lesssim\left|z_{1}-z_{2}\right|^{\alpha}
$$

for all $z_{1}, z_{2} \in D_{1}$.

(5.5) Remark. As stated in Proposition 4.5, if $D_{2}$ has smooth boundary, the integer $m$ in (5.2) can be chosen to be 1. Hence, in this case, $\alpha$ in Theorem 5.4 can be chosen to be $1 / 2$. Thus, in particular, one obtains Henkin's result [10, Theorem 1].

By applying the Theorem to $F$ and $F^{-1}$, one obtains

5.6. CoROllary. Let $F$ be a biholomorphic map between two bounded domains with piecewise smooth strictly pseudoconvex boundaries. Then $F$ extends to a homeomorphism between the closures of the domains.

6. Some open questions. (6.1) Theorem 3.2 is not the best possible statement. By considering the projections of the vector $v$ onto the holomorphic tangent spaces of the hypersurfaces which make up the boundary of $D$, one should obtain more precise estimates. Specifically, how do the precise results of Graham [6], [7] generalize to the domains considered in this paper?

(6.2) Proposition 4.3 is valid also for analytic polyhedra, but it is clearly false if $D$ is not holomorphically convex. For which other classes of domains does it hold? This problem is probably related to a question of Kohn [12, §7, III].

(6.3) As Henkin [10] has shown, a biholomorphic map between two domains with smooth $C^{2}$ strictly pseudoconvex boundaries is Hölder continuous of order $1 / 2$ (see also Remark 5.5). In view of Fefferman's result [4], this is probably not the best possible result. Specifically, is there a differentiable extension in case of $C^{2}$ boundaries?

(6.4) The proof of the continuous extension of biholomorphic maps given in this paper makes heavy use of the strict pseudoconvexity of the boundary. However, it is not at all clear, at least not to this author, that this condition is necessary. Thus, it is reasonable to ask: Is Corollary 5.6 valid for domains of holomorphy with smooth boundary?

\section{REFERENCES}

1. H. Behnke and F. Sommer, Theorie der analytischen Funktionen einer komplexen Veränderlichen, 2nd rev. ed., Die Grundlehren der math. Wissenschaften, Band 77, SpringerVerlag, Berlin, 1962. MR 26 \#5137.

2. H. Behnke and P. Thullen, Theorie der Funktionen mehrer komplexer Veränderlichen, 2nd rev. ed., Ergebnisse der Mathematik und ihrer Grenzgebiete, Band 51, SpringerVerlag, Berlin and New York, 1970. MR 42 \#6274.

3. K. Diederich, Das Randverhalten der Bergmanschen Kernfunktion und Metrik in streng pseudo-konvexen Gebieten, Math. Ann. 187 (1970), 9-36. MR 41 \#7149.

4. C. Fefferman, The Bergman kernel and biholomorphic mappings of pseudoconvex domains, Invent. Math. 26 (1974), 1-65. 
5. G. M. Goluzin, Geometric theory of functions of a complex variable, GITTL, Moscow, 1952; English transl., Transl. Math. Monographs, vol. 26, Amer. Math. Soc., Providence, R. I., 1969. MR 15, 112; 40 \#308.

6. I. Graham, Boundary behavior of the Carathéodory, Kobayashi, and Bergman metrics on strongly pseudoconvex domains in $\mathrm{C}^{n}$ with smooth boundary, Bull. Amer. Math. Soc. 79 (1973), 749-751. MR 48 \#2428.

7. I. Graham, Boundary behavior of the Carathéodory and Kobayashi metrics on strong. ly pseudoconvex domains in $\mathrm{C}^{n}$ with smooth boundary, Trans. Amer. Math. Soc. 207 (1975), 219-240.

8. R. C. Gunning and H. Rossi, Analytic functions of several complex variables, Prentice-Hall, Englewood Cliffs, N. J., 1965. MR 31 \#4927.

9. G. M. Henkin, Integral representations of functions holomorphic in strictly pseudo. convex domains, and some applications, Mat. Sb. 78 (120) (1969), 611-632 = Math. USSR 7 (1969), 597-616. MR 40 \#2902.

10. An analytic polyhedron is not holomorphically equivalent to a strictly pseudoconvex domain, Dokl. Akad. Nauk SSSR 210 (1973), 1026-1029 = Soviet Math. Dokl. 14 (1973), 858-862. MR 48 \#6467.

11. L. Hörmander, $L^{2}$ estimates and existence theorems for the $\bar{\partial}$ operator, Acta Math. 113 (1965), 89-152. MR 31 \#3691.

12. J. J. Kohn, Boundary behavior of $\bar{\partial}$ on weakly pseudo-convex manifolds of dimension two, J. Differential Geometry 6 (1972), 523-542. MR 48 \#727.

13. E. Ramírez de Arellano, Ein Divisionsproblem und Randintegraldarstellungen in der komplexen Analysis, Math. Ann. 184 (1969/70), 172-187. MR 42 \#4767.

14. R. M. Range and Y. T. Siu, Uniform estimates for the $\bar{\partial}$-equation on domains with piecewise smooth strictly pseudoconvex boundaries, Math. Ann. 206 (1973), 325-354.

15. H.-J. Reiffen, Die Carathéodorysche Distanz und ihre zugehörige Differentialmetrik, Math. Ann. 161 (1965), 315-324. MR 33 \#4325.

16. N. Vormoor, Topologische Fortsetzung biholomorpher Funktionen auf dem Rande bei beschränkten streng pseudokonvexen Gebieten im $\mathrm{C}^{n}$ mit $C^{\infty}$-Rand, Math. Ann. 204 (1973), 239-261.

\section{DEPARTMENT OF MATHEMATICS, STATE UNIVERSITY OF NEW YORK, AL-} BANY, NEW YORK 12222

Current address: Department of Mathematics, University of Washington, Seattle, Washington 98195 